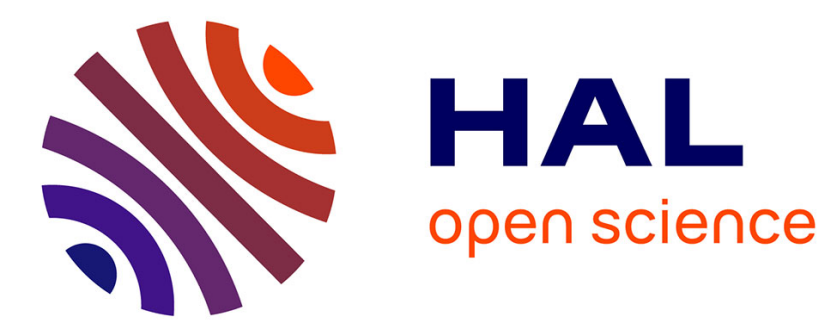

\title{
Étude des harmoniques de la luminance et des composantes wattées d'une cellule électroluminescente
}

\author{
D. Magnant, H. Payen de La Garanderie
}

\section{To cite this version:}

D. Magnant, H. Payen de La Garanderie. Étude des harmoniques de la luminance et des composantes wattées d'une cellule électroluminescente. Revue de Physique Appliquée, 1971, 6 (3), pp.361-363. 10.1051/rphysap:0197100603036100 . jpa-00243556

\section{HAL Id: jpa-00243556 https://hal.science/jpa-00243556}

Submitted on 1 Jan 1971

HAL is a multi-disciplinary open access archive for the deposit and dissemination of scientific research documents, whether they are published or not. The documents may come from teaching and research institutions in France or abroad, or from public or private research centers.
L'archive ouverte pluridisciplinaire HAL, est destinée au dépôt et à la diffusion de documents scientifiques de niveau recherche, publiés ou non, émanant des établissements d'enseignement et de recherche français ou étrangers, des laboratoires publics ou privés. 


\title{
ÉTUDE DES HARMONIQUES DE LA LUMINANCE ET DES COMPOSANTES WATTÉES D'UNE CELLULE ÉLECTROLUMINESCENTE
}

\author{
D. MAGNANT et H. PAYEN DE LA GARANDERIE
}

Laboratoire de Luminescence, Faculté des Sciences de Poitiers

(Reçu le 20 avril, révisé le 25 mars 1971)

\begin{abstract}
Résumé. - Un ordinateur nous a permis d'étudier les non-linéarités des phénomènes périodiques de l'électroluminescence. A partir des coefficients de Fourier nous avons pu retrouver les lois classiques de variation de la luminance. Nous avons pu également établir des relations entre la puissance absorbée et l'émission lumineuse.

Abstract. - We studied the nonlinear effects in an electroluminescent cell by mean of an IBM computer. The classical laws of dependance of the electroluminescence output can be found again from the Fourier's coefficients of these parameters. Relations can also be found between the power absorbed and the brightness of the emitted light.
\end{abstract}

L'excitation par une tension sinusoïdale d'une cellule électroluminescente (ici $\mathrm{ZnS}, \mathrm{Cu}, \mathrm{Cl}$ entre deux micas conducteurs) met en évidence un certain nombre de phénomènes périodiques : la luminance $L$, le courant $I$ traversant la cellule, la puissance électrique $P$ consommée, etc... L'étude des non-linéarités qui apparaissent automatiquement dans les variations de ces différents facteurs, nous a amené à mettre au point un programme d'analyse de Fourier sur ordinateur IBM 1620.

Programme de calcul. - Le programme de calcul est basé sur l'application d'une méthode de calcul numérique des coefficients d'une série de Fourier, ceci à partir de la considération du minimum de l'erreur quadratique moyenne [1].

La série de Fourier étant mise sous la forme :

$$
\mathrm{f}\left(t_{i}\right)=a_{0}+\sum_{k=1}^{p} a_{k} \cos k \omega t_{i}+\sum_{k=1}^{p} b_{k} \sin k \omega t_{i} .
$$

Les coefficients sont calculés à partir des formules :

$$
\begin{aligned}
& a_{0}=\frac{1}{n+1} \sum_{i=0}^{n} \mathrm{f}\left(t_{i}\right) \\
& a_{k}=\frac{2}{n+1} \sum_{i=0}^{n} \mathrm{f}\left(t_{i}\right) \cos k \omega t_{i} \\
& b_{k}=\frac{2}{n+1} \sum_{i=0}^{n} \mathrm{f}\left(t_{i}\right) \sin k \omega t_{i} .
\end{aligned}
$$

A partir des pointés sur un oscillogramme nous pouvons connaître le pourcentage d'harmonique et par un programme annexe les modules et les phases des différentes harmoniques. On peut ainsi effectuer une reconstitution graphique des courbes étudiées.
Application à l'étude de l'onde périodique de luminance $L$. - Dans la suite de l'article nous désignerons par $H_{i x}$ l'harmonique d'ordre $i$ de la quantité $x$, ainsi $H_{2 L}$ sera l'harmonique d'ordre 2 de la luminance $L$.

L'harmonique $H_{2 L}$ représente le pic principal et $H_{4 L}$ traduit le pic secondaire. Une étude des courbes de variations de $H_{2 L}$ et $H_{4 L}$ en fonction de la tension

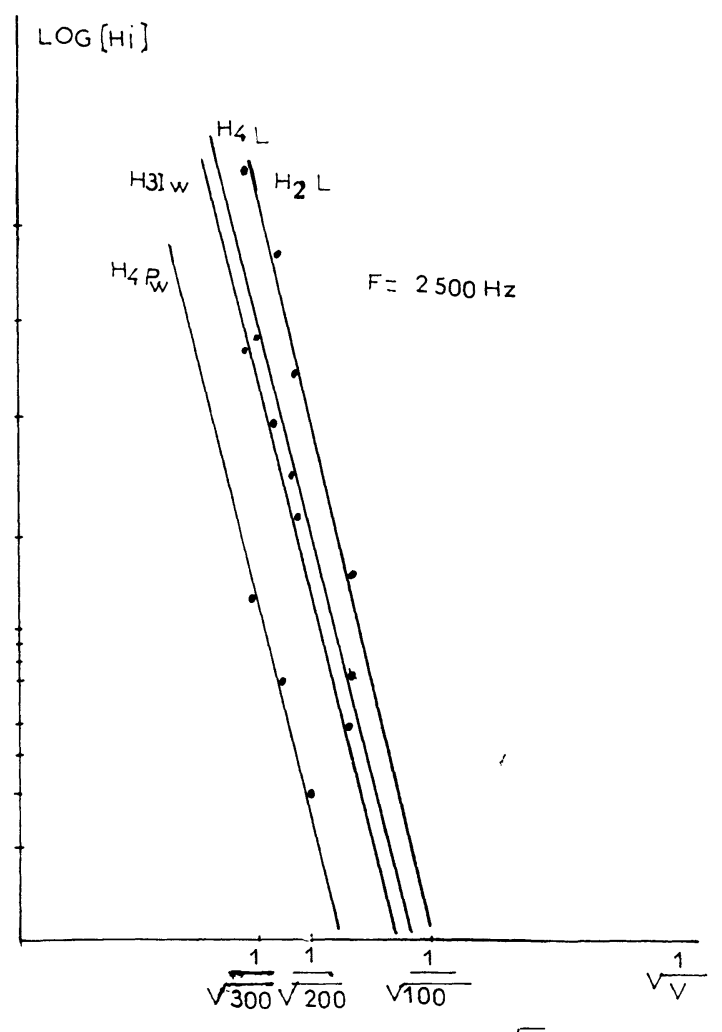

FIG. 1. $-\log \left(H_{i x}\right)=\mathrm{f}(1 / \sqrt{V})$. 
(Fig. 1) nous montre que ces coefficients suivent bien la loi de Destriau en $\mathrm{e}^{-\boldsymbol{B} / \sqrt{V}}$.

Le fondamental $H_{1 L}$, seul terme impair, met en évidence la dissymétrie qui existe entre les deux pics primaires au cours d'une période.

D'après Destriau et Yvey [2], si $a$ et $d$ sont les hauteurs respectives des 2 pics primaires le facteur de modulation $m$ s'écrit :

$$
m=2 \frac{a-d}{a+d} .
$$

On a alors :

$$
\frac{H_{1 L}}{H_{2 L}} \simeq 2 \mathrm{~m}
$$

L'étude de ce rapport permet aussi de connaître les inversions qui peuvent se produire dans la dissymétrie.

Notre méthode présente l'intérêt de permettre une étude précise même lorsque le pic secondaire n'est pas marqué par rapport au principal ; ceci étant souvent le cas pour une étude en température.

Application à l'étude des composantes wattées $\boldsymbol{I}_{\mathrm{w}}$ et $\boldsymbol{P}_{\mathrm{w}}$. - Pour connaître la composante wattée du courant traversant la cellule nous avons employé la méthode du pont différentiel [3].

Le tracé des caractéristiques courant-tension des $H_{i \text { I w }}$ fait apparaître, sur le fondamental, le caractère redresseur des cellules électroluminescentes. Le fait que le spectre de $I_{\mathrm{w}}$ ne soit composé que des termes impairs nous permet de préciser d'après [4] que l'on a affaire à des redresseurs non parfaits (caractéristiques franchement dissymétriques par rapport à la résistance moyenne).

Une étude faite sur différents types de cellules électroluminescentes nous montre que dans tous les cas l'harmonique $H_{3 I w}$ est prépondérante par rapport aux harmoniques autres que le fondamental. Nous allons voir comment cet harmonique semble lié au phénomène d'électroluminescence lui-même.

En simplifiant à l'extrême on peut dire que la lumière (termes en $2 \omega t$ et $4 \omega t$ ) a pour origine l'accélération par le champ (terme en $\omega t$ ) d'électrons qui viennent ioniser les centres luminogènes. D'après des considérations trigonométriques simples pour que le produit du terme champ en $\omega t$ par le terme courant conduise à des termes en $2 \omega t$ et $4 \omega t$ il faut que ce dernier soit en $3 \omega t$.

On en déduit facilement que c'est le terme en $4 \omega t$ de la puissance wattée $P_{\mathrm{w}}$ qui pourra être mis en relation avec la luminance.

Nous voyons d'après la courbe $n^{0} 1$ que les quantités $H_{3 I w}$ et $H_{4 P w}$ vérifient la loi de Destriau avec une pente identique à celle de la luminance. D'autre part en fonction de la fréquence (courbes $n^{\circ} 2,3$ et 4 ) les maximums des trois quantités se produisent pour une fréquence voisine de $5000 \mathrm{~Hz}$.

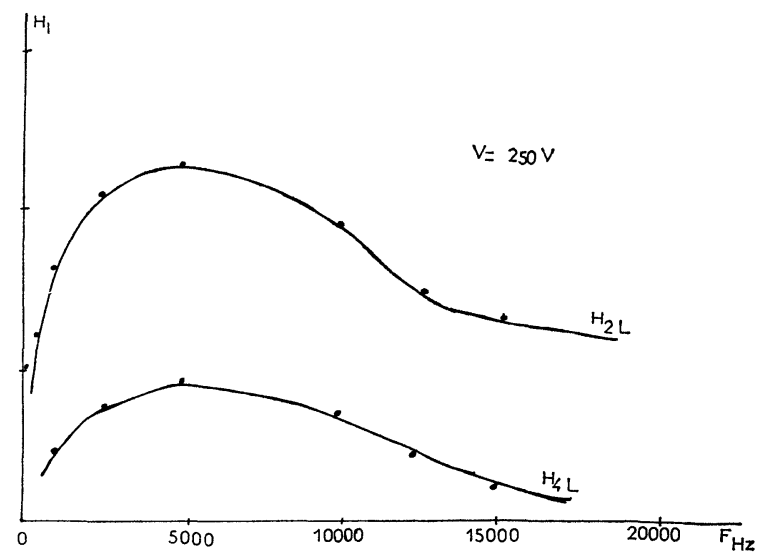

FIG. 2. $-H_{i l}=\mathrm{f}(F)$.

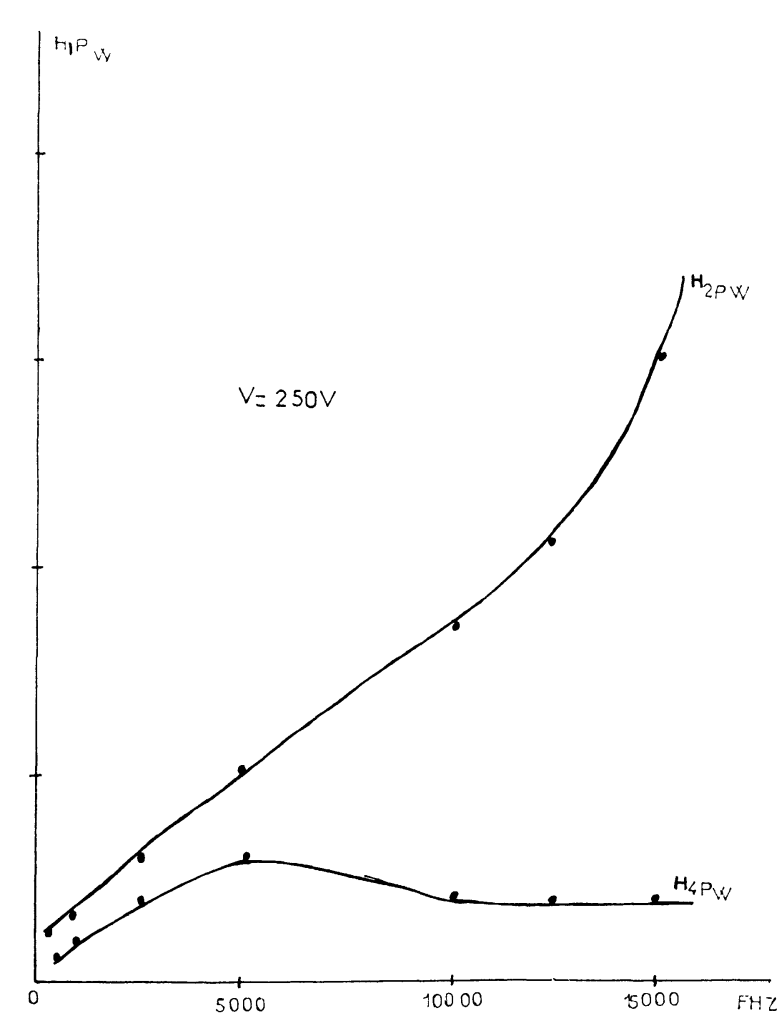

FIG. 3. $-H_{i I \mathrm{w}}=\mathrm{f}(F)$.

Des relations de phases confirment ces relations en module. On peut déduire que, en première approximation, que l'harmonique d'ordre $3 \mathrm{du}$ courant watté $I_{\mathrm{w}}$ traduit le mouvement des électrons tête de chaîne qui viennent ioniser par choc, après avoir été accélérés par le champ, les centres luminogènes.

Nous avons tracé sur la figure 5 les positions relatives moyennes au cours d'une période des quantités $V$, $H_{4 P_{\mathrm{w}}}, H_{2 L}$ et $H_{4 L}$. Dans [4] où l'on considère globalement $L, I$ et $P_{\mathrm{w}}$, les maximums de toutes ces quantités se produisent dans l'ordre dans lequel elles ont été citées. Nous voyons qu'ici chaque maximum de lumière, pic primaire ou secondaire, est précédé par un maximum de puissance wattée absorbée par le 


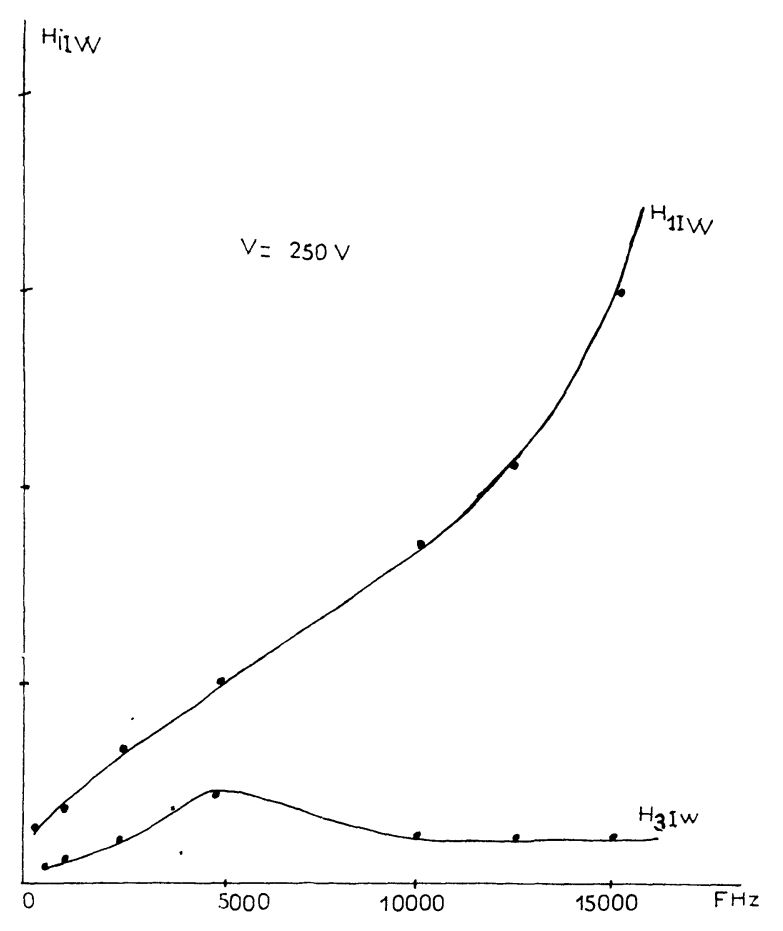

FIG. 4. $-H_{i P \mathrm{w}}=\mathrm{f}(F)$.

produit. Nous pouvons donc faire un rapprochement entre la puissance absorbée et celle qui est restituée.

Les relations de phase, et surtout les modifications de ces relations pour une tension croissante, par exemple, montrent bien les relations existant entre ces trois quantités. Nous retrouverons en effet la simultanéité des phénomènes d'absorption d'énergie et de réémission de lumière.

Des études comparées des variations en fonction de la tension du rendement de la luminance et de

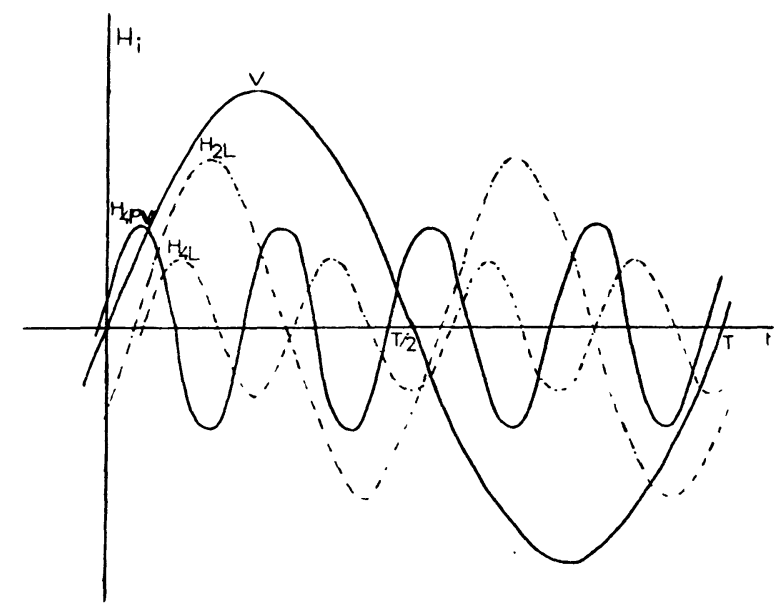

FIG. 5. - Positions relatives, au cours d'une période des courbes $V, H_{4 P \mathrm{w}}, H_{2 L}$ et $H_{4 L}$.

l'harmonique d'ordre 4 de la puissance wattée nous permettent de dire que $H_{4 P_{\mathrm{w}}}$ est proportionnel à la partie de l'énergie électrique qui est transformée en lumière. Alors que l'harmonique $H_{2 P_{w}}$ dont les maximums apparaissent après ceux de lumière traduit les pertes dans le diélectrique.

Conclusion. - Sans donner une véritable signification physique aux harmoniques des différents paramètres de l'électroluminescence, on peut donc, par cette première étude, déceler quels sont les harmoniques intéressants à étudier et établir des relations entre ceux-ci. Nous pensons donc employer cette même méthode par l'étude de phénomènes identiques mais cette fois sous irradiation U. V. et comparer ces résultats avec ceux obtenus pour les variations de la constante diélectrique complexe.

\section{Bibliographie}

[1] Angot (A), Compléments de Mathématiques.

[2] Destriau et Ivey, Proc. Rw. opth. IRE, 1955, 1911.

[3] Georgobiani (A. N.), L'Vova (E. YU.) and
Fock (M. V.), Opt. Spectry, U. S. A., 1963, 15, 142-3.

[4] Vitold Bellevitch, Théorie des circuits non linéaires en régime alternatif 